\title{
Red and processed meat consumption and nutritional adequacy among British adults
}

\author{
D.A. Hobbs ${ }^{1,2,3}$, J.A. Lovegrove ${ }^{1,2,3}$ and D.I. Givens $s^{2,3}$ \\ ${ }^{1}$ Hugh Sinclair Unit of Human Nutrition, Department of Food and Nutritional Sciences, \\ ${ }^{2}$ Institute for Food, Nutrition and Health and \\ ${ }^{3}$ Institute for Cardiovascular and Metabolic Research, University of Reading, Reading, RG6 6AP, UK.
}

Red meat is a good source of a number of nutrients such as iron, zinc and B vitamins ${ }^{(1)}$. However, consumption of red and processed meat has been associated with unfavourable health outcomes including an increased risk of colon cancer ${ }^{(2)}$. The aim of this study was to examine the association between diets with varying levels of red meat (RM), processed red meat (PRM) and total red and processed meat (TRPM) with nutritional adequacy in adults.

A secondary analysis was conducted using data from 1068 adults aged 19-64 years from the National Diet and Nutrition Survey (NDNS) years 1-4 (2008/09-2011/12) $)^{(3)}$. Adults (221 men and 517 women) were classified into tertiles according to TRPM consumption, with tertile 1 (T1, 0-41 g/day) and tertile 3 (T3, 87-344 g/day) representing diets with the lowest and highest intake of TRPM, respectively. Intakes were obtained from 4-day diet diaries. ANCOVA was used to determine statistically significant differences between tertiles of TRPM, RM and PRM consumption and macro-and micro-nutrient intakes, plasma ferritin and haemoglobin $(\mathrm{Hb})$ concentrations (biomarkers of iron status) controlling for age, sex, total energy intake (kJ), BMI, social class and smoking status. Participants with fasted blood glucose above $7 \mathrm{mmol} / \mathrm{L}$ or taking medicines known to affect blood analytes were excluded from the statistical analysis.

The mean TRPM, PRM and RM consumption was 70.3 (SD 54.0), 36.6 (SD 37.4) and 33.7 (SD 36.3) g/day, respectively. Furthermore, $56 \%$ of participants consumed less than SACN recommendations $(<70 \mathrm{~g} / \text { day })^{(1)}$ for TRPM. Intakes of saturated fat, MUFA, trans-fat, thiamine, haem iron (dietary) and plasma $\mathrm{Hb}$ concentration were significantly higher in diets of participants in the high TRPM, RM and PRM tertiles (T3), respectively (all $P<0 \cdot 05$ ). In addition, protein, niacin, iron and zinc intakes were significantly higher for T3 compared with T1 for TRPM and RM, respectively (all $P<0.05)$. Furthermore, total fat and sodium intakes were higher for T3 compared with T1 for TRPM and PRM, respectively (all $P$-trend $=0 \cdot 0001$ ). Carbohydrate, vitamin E, biotin and manganese intakes were significantly lower for TRPM, RM and PRM, respectively (all $P<0.05$ ). Calcium and iodine intakes were significantly lower for TRPM (calcium: $P$-trend $=0.009$; iodine: $P$-trend $=0.0001)$ and RM (calcium: $P$-trend $=0.001$; iodine: $P$-trend $=0.0001)$, respectively. Vitamin B12 $(P$-trend $=0.019)$, iron $(P$-trend $=0.002)$, non-haem iron $(P$-trend $=0.0001)$ and selenium $(P$-trend $=0.006)$ intakes were significantly lower for T3 compared with T1 for PRM. Moreover, the percentage of participants not meeting the Lower Recommended Nutrient Intake (LRNI) were lower for high TRPM (T3) compared with low TRPM consumers (T1) for iron (27 vs. $3 \%)$, zinc (11 vs. $1 \%$ ), selenium (49 vs. $11 \%$ ) and vitamin B6 (13 vs. $1 \%)$.

This study suggests that around half $(56 \%)$ of the UK population has TRPM intakes that are in line with current recommendations ${ }^{(1)}$. Diets containing high RM $(44-225 \mathrm{~g} / \mathrm{d})$ have an overall better nutrient profile compared with diets containing high PRM (42-284 g/d). Furthermore, dietary patterns that contain low TRPM (0-41 g/day) may have implications for iron, zinc, vitamin B6 and selenium intakes and status in British adults.

This work was supported by the Agriculture and Horticulture Development Board (AHDB).

1. Scientific Advisory Committee on Nutrition (SACN) (2011) Iron and Health Report. https://www.gov.uk/government/publications/sacn-iron-andhealth-report (accessed August 2017).

2. Vieira AR, Abar L, Chan DSM et al. (2017) Ann Oncol 8, 1788-1802.

3. Bates B, Lennox A, Prentice A et al. (2017) National Diet and Nutrition Survey (NDNS) - results from years 1-4 (combined) of the rolling programme (2008/2009-2011/2012). https://www.gov.uk/government/uploads/system/uploads/attachment_data/file/594361/NDNS_Y1_to_4_UK_ report_full_text_revised_February_2017.pdf (accessed August 2017). 